\title{
Antenna Sensors Prepared by Laser Direct Writing Based on Graphene Hybrid Materials
}

\author{
Akira Watanabe ${ }^{1}$, Ashiqur Rahman ${ }^{1,2}$, Jinguang Cai ${ }^{3}$, and \\ Mohammod Aminuzzaman ${ }^{4}$ \\ ${ }^{1}$ Institute of Multidisciplinary Research for Advanced Materials, Tohoku University, \\ 2-1-1 Katahira, Aoba-ku, Sendai 980-8577, Japan \\ ${ }^{2}$ Department of Electrical and Electronics Engineering, Faculty of Engineering, \\ American International University Bangladesh, \\ 408/1, Kuratoli Road, Kuril Dhaka-1229, Bangladesh \\ ${ }^{3}$ Institute of Materials, China Academy of Engineering Physics, Jiangyou 621908, \\ Sichuan, P. R. China \\ ${ }^{4}$ Department of Chemical Science, Faculty of Science, Universiti Tunku Abdul Rahman \\ (UTAR), Perak Campus, Jalan Universiti, Bandar Barat, 31900 Kampar, Perak D. R., Malaysia \\ *akira.watanabe.c6@tohoku.ac.jp
}

Formation of an antenna-type sensor by laser direct writing based on graphene hybrid materials for humidity and chemical sensing properties was studied. A meander line shaped dipole antenna was prepared by laser direct writing on a graphene oxide (GO)$\mathrm{CuO}$ NRs hybrid film. Resonance peaks were observed at around 4.7 and $5.89 \mathrm{GHz}$ showing the dependence on relative humidity (RH). The humidity increase from RH38 to RH100 caused a resonance peak shift from 5.89 to $5.85 \mathrm{GHz}$ accompanying the increase of return loss from -17.76 to $-34.07 \mathrm{GHz}$. A spiral antenna sensor consisting of GO-coated copper-carbon hybrid showed the resonance band shift depending on the dielectric constant of a chemical compound.

Keywords: Graphene oxide, Reduced graphene oxide, Laser direct writing, $\mathrm{CuO}$ nanorods, Antenna-type sensor, Humidity sensor, Chemical sensor

\section{Introduction}

The IoT (internet of things) is based on sensor network consisting of a huge number of sensors and portable devices which are connected each other on internet. The progress of various kinds of sensors and portable devices is an important issue in various kinds of fields [1-3]. The innovation of sensor manufacturing process both in material and fabrication technique is necessary to reduce the cost of them. The printed electronics is one of the candidates for the innovative manufacturing. The contribution of laser processing in printed electronics is expected because of the compatibility with on-demand manufacturing and role-to-role processing. Graphene is expected to play an important role in various fields including semiconductor materials, displays and filters because of a high conductivity and mechanical stability. Graphene oxide (GO) is one of graphene family nanomaterials, which can be applied to solution processes because of dispersibility in water and polar solvents. The dispersibility is caused by oxygen-containing groups on the graphene plane. Although GO is almost insulator, the reduction of GO gives a conductive reduced graphene oxide (rGO). In previous papers, we have reported the laser-induced reduction of $\mathrm{GO}$ to $\mathrm{rGO}$ and the device applications [4-7]. An interdigitated electrode of $\mathrm{rGO} / \mathrm{GO} / \mathrm{rGO}$ structure was prepared by laser direct writing on a GO-coated flexible polymer substrate for application as a flexible and high-sensitive humidity sensor, where the sensing mechanism is based on the enhancement of ionic conductivity by water trapping into the GO layer by oxygen containing groups [6]. The sensor also responded to protonic molecules such as $\mathrm{MeOH}$ and 
EtOH although the sensitivity was much lower than that for water. Because the sensing was conducted by detection of the DC voltage change induced by the conductivity and capacitance changes, the selective detection for protonic molecules with different chemical structure was difficult. In this paper, we challenge an antenna-type sensor based on the changes in high frequency characteristics, where multi-factor detection is possible, for example monitoring resonance frequency and return loss signal using a vector network analyzer (VNA). The laser direct writing is an on-demand manufacturing process, which enables a firstproduction evaluation system for an antenna device. Two kinds of antenna type chemical sensors were developed based on the laser written conductive film.

\section{Experimental}

The $20 \mathrm{mg}$ of few-layer GO powders (Beijing Ding Sheng Xiong Di Technology Co., Ltd) was dispersed in the water $(5 \mathrm{~g})$ to form a GO dispersion. After the ultrasonication in a water bath for $5 \mathrm{~min}$, a gel-like solution was formed. The $\mathrm{CuO}$ nanorods (NRs) were synthesized using a plant leaf extract as similar manner reported in previous papers $[8,9]$. The laser source was a continuous-wave (CW) 445 $\mathrm{nm}$ blue semiconductor laser $(3 \mathrm{~W})$. The laser beam was scanned by a galvano-scanner through an $\mathrm{f}$ $\theta$ lens with a focus length of $110 \mathrm{~mm}$. A GO-CuO NRs hybrid-coated PET substrate with a size of 20 $\times 30 \mathrm{~mm}$ was set in a box with a glass window and then a $445 \mathrm{~nm}$ laser beam was scanned under Ar gas flow rate of $40 \mathrm{sccm}$. A meander line antenna pattern was drawn on a GO-CuO NRs hybrid film by using a laser marking software (EzCAD, BJJCZ). Return loss spectra were observed by a VNA (TTR506A, Tektronix).

\section{Results and discussion}

Figure 1a shows the optical microscope image of laser scanned hybrid films consisting of GO and $\mathrm{CuO}$ NRs with a laser power of $500 \mathrm{~mW}$, a scan rate of $100 \mathrm{~mm} / \mathrm{s}$, and $60 \mu \mathrm{m}$ line spacing. The grains of copper formed by laser-induced reduction enhanced with GO were observed [7]. The surface resistivity was determined to be $199 \mathrm{k} \Omega / \mathrm{sq}$ by 4-probe method. The overall view of a laser-written meander line antenna is shown in Fig. 1b. The antenna pattern was connected to a coaxial cable and SMA connector. The return loss spectra of the antenna sensor are shown in Fig. 2. Resonance peaks were observed at around 4.7 and $5.89 \mathrm{GHz}$ showing the dependence on relative humidity (RH). The humidity increase from RH38 to RH100 caused a resonance peak shift from 5.89 to $5.85 \mathrm{GHz}$ accompanying the increase of return loss from 17.76 to $-34.07 \mathrm{GHz}$.
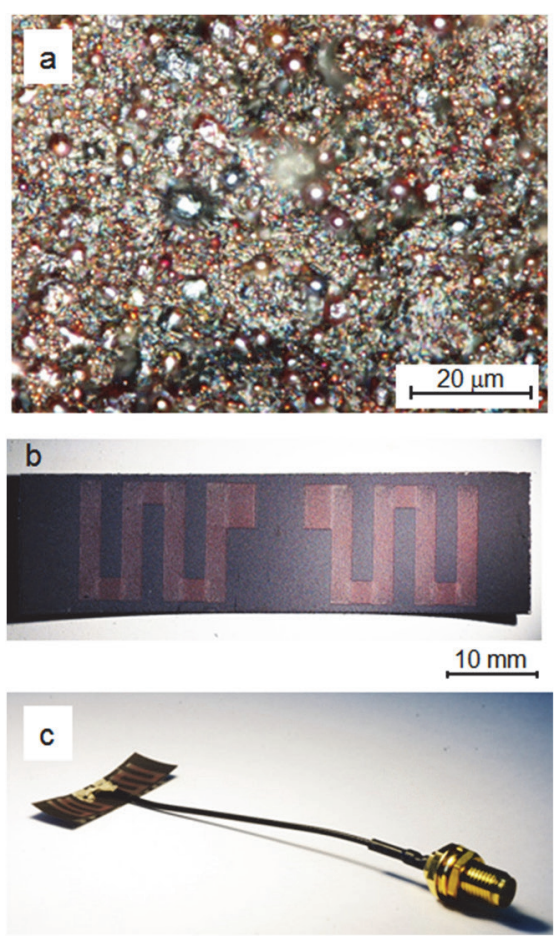

Fig. 1. Optical microscope image of laser scanned hybrid film of $\mathrm{GO}$ and $\mathrm{CuO}$ NRs (a) and digital photographs of a meander line antenna (b) and antenna-type sensor (c).
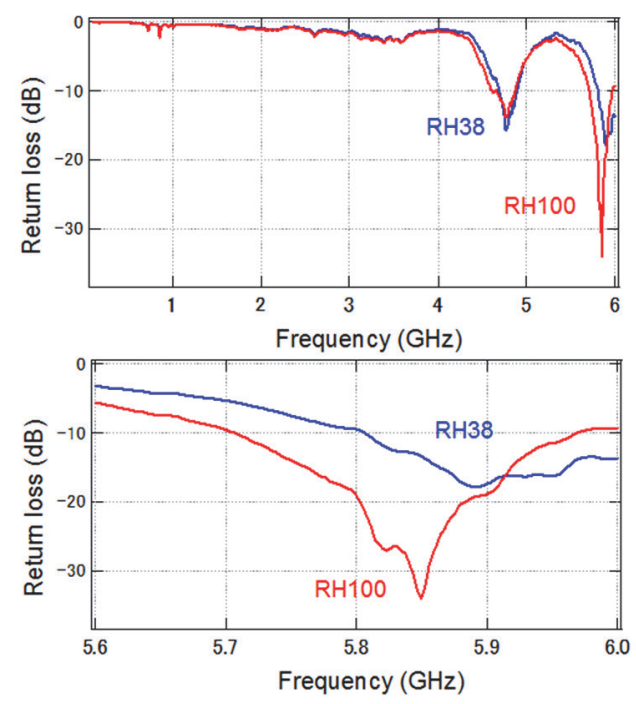

Fig. 2. Humidity dependence of return loss spectra of an antenna-type sensor. 
The other type of antenna was prepared to enhance the sensitivity. The laser direct writing process is illustrated in Fig. 3. A spiral antenna pattern consisting of a conductive carbon layer (Fig. 4a) was prepared on a polyimide (PI) film by laser direct writing using a $445 \mathrm{~nm}$ galvano-scanning system. The surface resistivity of the carbon pattern was ca. $100 \Omega / \mathrm{sq}$, which enabled the copper electroplating on the spiral antenna carbon pattern (Fig. 4b). The drop-casting of GO was conducted on

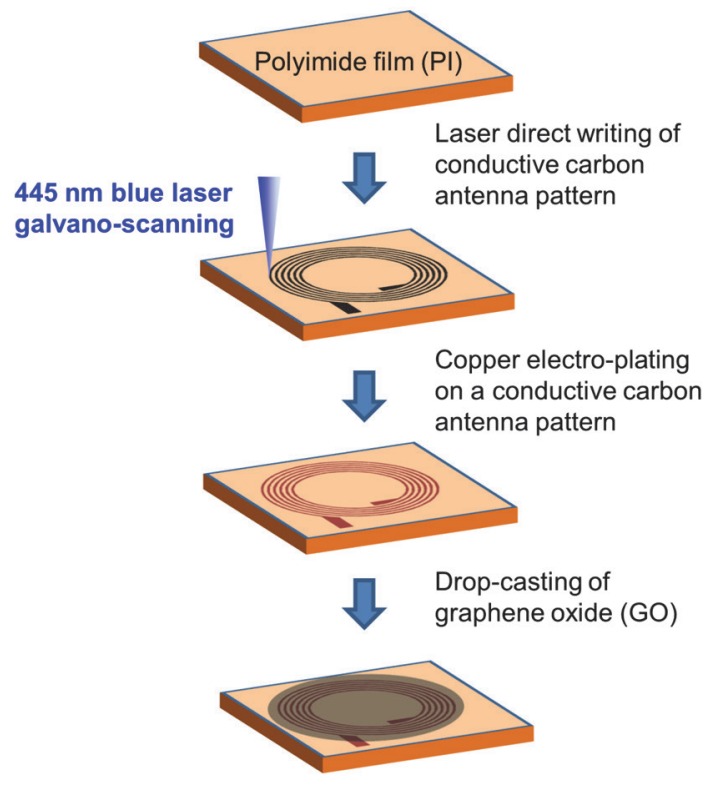

Fig. 3. Formation of a GO-coated copper-carbon hybrid antenna by laser direct writing and copper electroplating.

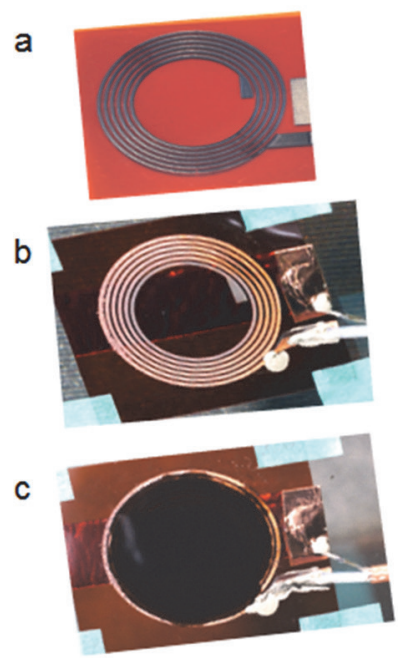

Fig. 4. Photographs of (a) laser written spiral antenna consisting of a conductive carbon layer on PI film, (b) copper-carbon hybrid antenna by electro-plating, (c) GO-coated copper-carbon hybrid antenna. the copper-carbon hybrid antenna (Fig. 4c). Return loss spectral changes of GO-coated copper-carbon hybrid antenna during humidity increase are shown in Fig. 5. The GO-coated copper-carbon hybrid antenna showed two characteristic resonance bands at around 2.17 and $5.36 \mathrm{GHz}$. The shift of the resonance band at around $5.36 \mathrm{GHz}$ to the lower frequencies was observed with increasing in relative humidity. The shift to the lower frequencies and increase of the return loss at the higher humidity conditions were similar with the characteristics of a meander line antenna as shown in Fig. 2. Such phenomenon must be caused by the changes of dielectric properties of GO under a humidity condition. On the other hand, the resonance band at around $2.17 \mathrm{GHz}$ showed the decrease of the return loss although the direction of the shift to the lower frequencies was same as $5.36 \mathrm{GHz}$ band. Figure 6 shows return loss spectral changes of GO-coated copper-carbon hybrid antenna during humidity decrease. The shift to the higher frequencies and return loss decrease were observed, which were almost reversible changes for the increase condition. The additional feature of the resonance band at the higher than $5.36 \mathrm{GHz}$ is the remarkable increase of the return loss of the other resonance band at around

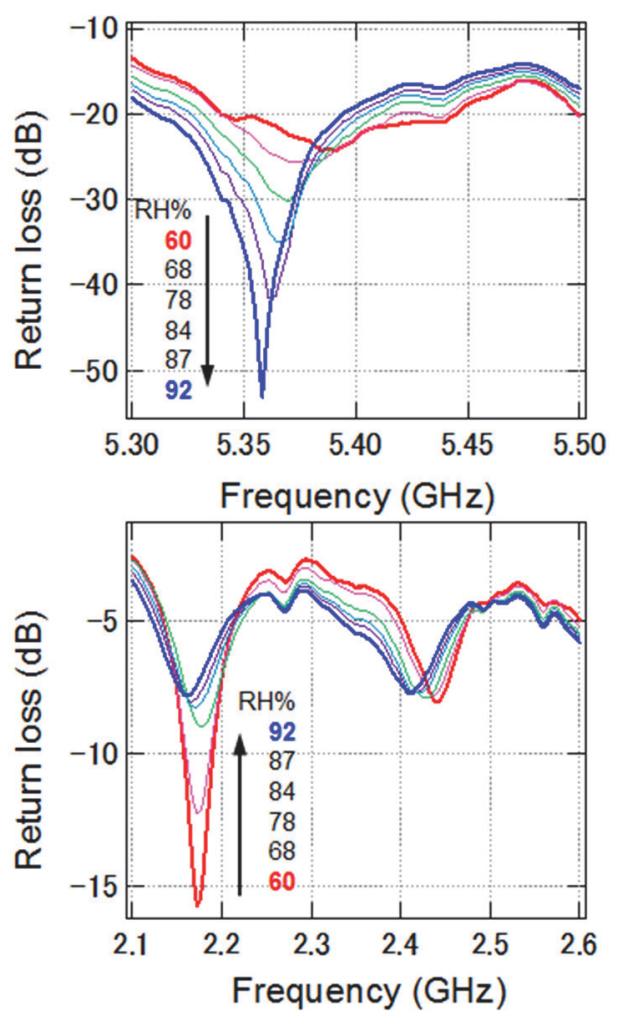

Fig. 5. Return loss spectral changes of GO-coated copper-carbon hybrid antenna during humidity increase. 
$5.43 \mathrm{GHz}$ during humidity decrease. A clear isosbestic point was observed at around $5.39 \mathrm{GHz}$ between 5.36 and 5.43 resonance bands. On the other hand, the remarkable feature of the resonance bands in the range of 2.1 to $2.6 \mathrm{GHz}$ was continuous shift to the higher frequencies with decreasing in humidity. The microwave in the range from 3 to 30 $\mathrm{GHz}$ is called as SHF (Super High Frequency), where the frequency response of dielectric mechanism is based on the dipole motion and reorientation induced by microwave irradiation. In the application of an antenna-type sensor to a chemical sensor which can detect the difference of chemical structure, the higher frequency resonance bands in SHF region is suitable for the chemical sensing. The dependence of return loss of GOcoated copper-carbon hybrid antenna on relative humidity for 5.36 and $5.43 \mathrm{GHz}$ resonance bands are shown in Fig. 7, where the relative humidity RH was determined by a commercial sensor. A good correlation between return loss and RH suggested that the antenna-type sensor can work as a humidity sensor.

Figure 8 shows the return loss spectra of GOcoated copper-carbon hybrid antenna for various

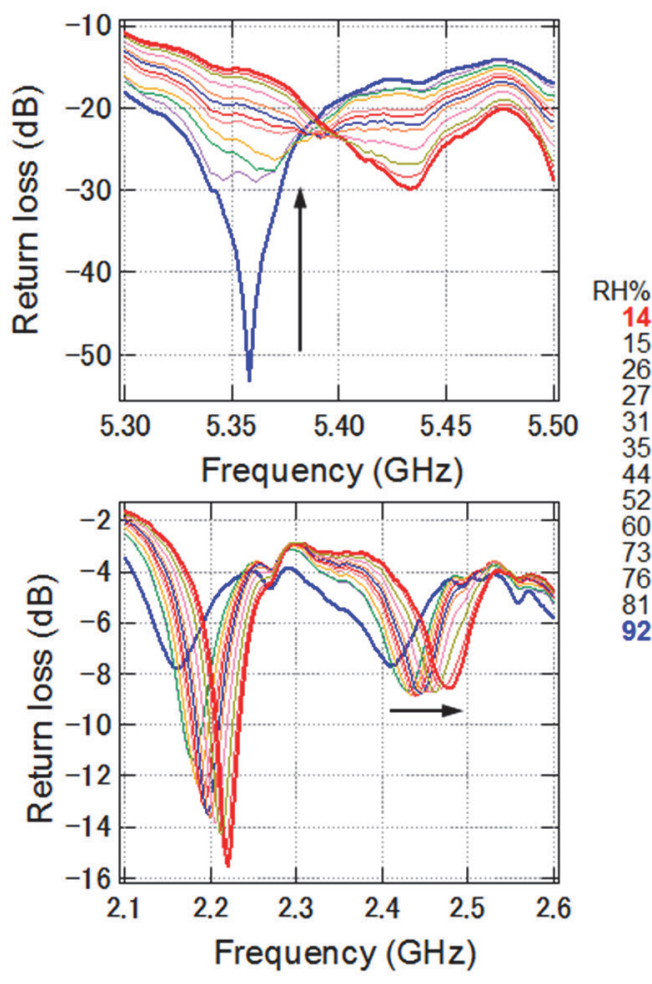

Fig. 6. Return loss spectral changes of GO-coated copper-carbon hybrid spiral antenna during humidity decrease. chemical molecules such as water, $\mathrm{MeOH}, \mathrm{EtOH}$, and $n$-hexane, which have dielectric constants of 80.4 32.6, 24.3, and 1.9, respectively. The measurements were carried out under saturated vapor conditions by setting the antenna sensor in a closed container with a chemical compound. The chemical molecule with the higher dielectric constants showed the lower resonance frequencies
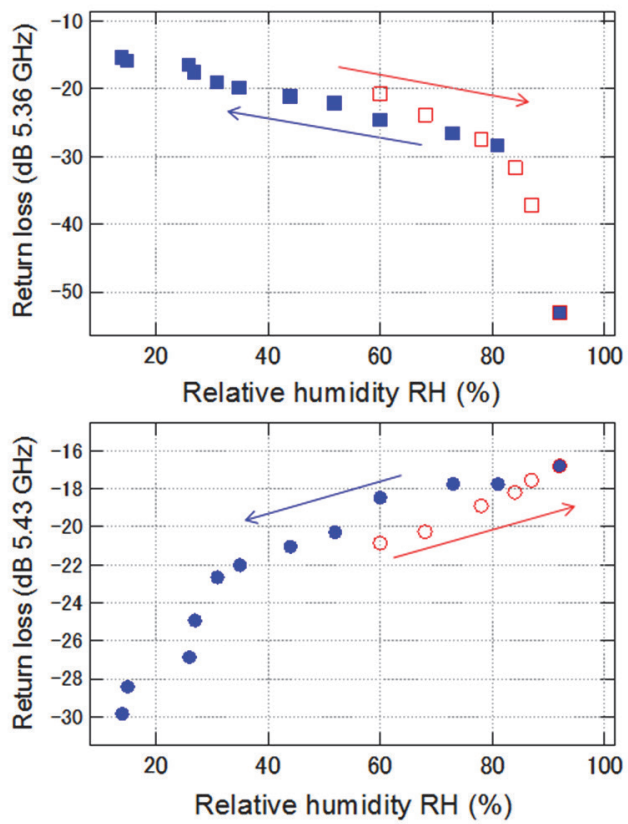

Fig. 7. Dependence of return loss of GO-coated copper-carbon hybrid spiral antenna on relative humidity.
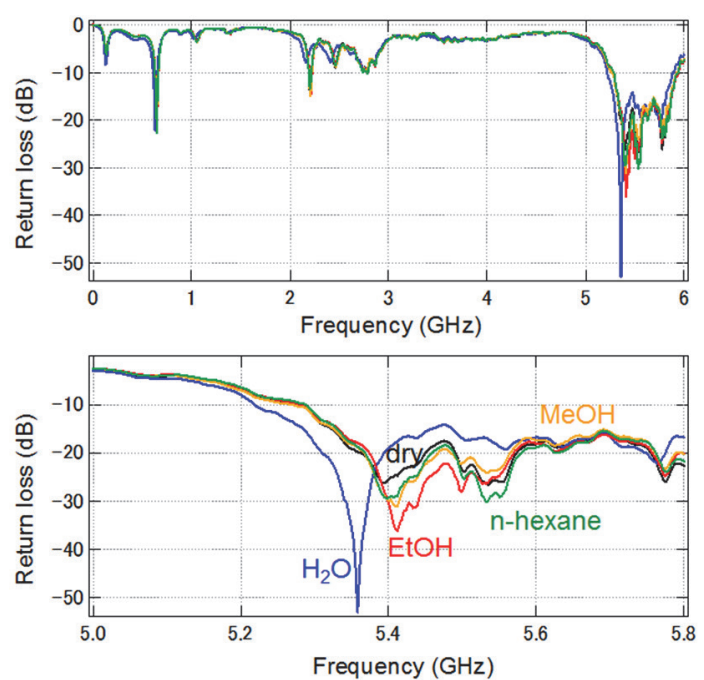

Fig. 8. Return loss spectra of GO-coated coppercarbon hybrid spiral antenna for various chemical molecules. 
as shown in Fig. 8. The return loss was higher with the higher dielectrics.

The characteristic resonance frequency depending on the dielectric constant of chemical compound can be applied to a chemical sensor based on frequency changes of an antenna-type sensor.

\section{Conclusion}

Antenna-type sensors consisting of graphene hybrid materials were prepared by laser direct writing. The shift of resonance band to the lower frequencies and increase of return loss were observed under a high humidity condition, which can be applied to a humidity sensor. A spiral antenna sensor consisting of GO-coated copper-carbon hybrid showed the resonance band shift depending on the dielectric constant of a chemical.

\section{Acknowledgements}

This work was partially supported by JSPS KAKENHI Grant Number JP15H04132, JSPS KAKENHI Grant Number JP19H05356, National Natural Science Foundation of China (No. 21603201), and Institute of Materials, China
Academy of Engineering Physics (item no. TP02201303).

\section{References}

1. S. Kaminaga, Sensor. Mater, 30 (2018) 723.

2. A. Zanella, N. Bui, A. Castellani, L. Vangelista, and M. Zorzi, IEEE Internet Things, 1 (2014) 22.

3. S. C. Mukhopadhyay, IEEE Sensors, 15 (2015) 1321.

4. J. Cai, C. Lv, and A. Watanabe, IEEE-NANO DOI: 10.1109/NANO.2016. 7751433 (2016).

5. A. Watanabe, J. Cai, S. Ogawa, E. Aoyagi, and S. Ito, J. Photopolym. Sci. Technol., 31 (2018) 447.

6. J. Cai, C. Lv, E. Aoyagi, S. Ogawa, and A. Watanabe, ACS Appl. Mater. Interfaces, 10 (2018) 23987.

7. A. Watanabe, M. Aminuzzaman, J. Cai, Md. Akhtaruzzaman, S. Ogawa, E. Aoyagi, and S. Ito, J. Photopolym. Sci. Technol., 32 (2019) 223.

8. M. Aminuzzaman, L. M. Kei, and W. H. Liang, AIP Conf. Proc., 1828 (2017) 020016.

9. M. Aminuzzaman, P-S. Ng, W-S. Goh, S. Ogawa, and A. Watanabe, Inorg. Nano-met. Chem., 49 (2019) 401. 